\title{
Rapid Combinatorial Screening of Inkjet-printed Alkyl-ammonium Cations in Perovskite Solar Cells
}

Monojit Bag ${ }^{1,2}$, Ziwen Jiang ${ }^{1}$, Lawrence A. Renna, Seung Pyo Jeong, Vincent M. Rotello* and D.

Venkataraman*

Department of Chemistry, University of Massachusetts Amherst, 710 North Pleasant Street, Amherst, MA

01003, United States

* Corresponding authors.

E-mail addresses:dv@chem.umass.edu (D.Venkataraman), rotello@chem.umass.edu (V.M. Rotello).

${ }^{1}$ These authors contributed equally to this work.

${ }^{2}$ Present address: Department of Chemical Physics, Lund University, Lund 22241, Sweden

\section{Abstract}

Herein we demonstrate that efficient perovskite solar cells can be fabricated by a desktop multi-channel inkjet printer. In this approach, different alkyl-ammonium counterions were loaded separately into the printer cartridges and deposited onto $\mathrm{PbI}_{2}$ coated substrates. Structural as well as electronic properties of perovskite solar cells can be tuned by varying the composition ratio of multiple cations in-situ through the RGB color codes of the multi-channel inkjet printer. This roll-to-roll compatible technique provides rapid and facile screening of multiple and mixed counterion perovskite solar cell systems with high reproducibility, potentially leading to possibilities of high-throughput screening technique in future perovskite solar cell fabrication.

\section{Key words}

Perovskite; Solar cell; Multi-channel inkjet printing; Combinatorial screening.

\section{Introduction}

Organic-inorganic hybrid perovskite solar cells [1-6] are among the most attractive alternatives in the area of renewable energy research due to their high efficiency [5, 7], flexibility [8], and low-cost solution processability [8]. Perovskite solar cells efficiencies have been optimized using solvent processing [9], device architecture, and interface engineering [7, 10, 11]. However, tuning the active layer material composition for increased stability and efficiency has largely been overlooked. Typically, the active layers of perovskite solar cells are fabricated using lead iodide $\left(\mathrm{PbI}_{2}\right)$ and 
methylammonium iodide $\left(\mathrm{CH}_{3} \mathrm{NH}_{3} \mathrm{I}\right)$. In the perovskite structure, methylammonium $\left(\mathrm{CH}_{3} \mathrm{NH}_{3}{ }^{+}, \mathrm{MA}\right)$ cations occupy the void space created by vertex-sharing $\mathrm{PbI}_{6}$ octahedra. The optical properties, band structure and electronic properties of organic/inorganic hybrid perovskite materials are correlated to the size of the organic cations. Cation size affects the Pb-I-Pb bond length and angle [12], with larger cations normally resulting in 2D perovskites with a wider band gap [13]. Recently formamidinium $\left(\mathrm{HN}=\mathrm{CHNH}_{3}{ }^{+}, \mathrm{FA}\right)[14-16]$ cations have been introduced instead of methyl ammonium to fabricate efficient perovskite solar cells. The sizes of both MA and FA cations meet the required size requirements (based on the Goldschmidt tolerance factor) [17] for the formation of a 3D perovskite. The advantages of using FA cations instead of MA include less hysteresis in the J-V curves due to low dipole moments [18], wider absorption [19] and improved dark stability [15, 16]. Typically, perovskite solar cells containing FA-only cation show lower power conversion efficiency (PCE) than MA-only device due to weak near-IR absorption [16].

The structural and electronic properties of perovskite materials can also be tuned by changing the composition of multiple organic cations. Several groups have tried combination of two-cation system for better performance of perovskite solar cells; significant improvement in PCE is observed when two of these cations (MA/FA) are mixed in certain ratio, albeit the ratio used by the two groups to fabricate high-efficiency devices are contradictory $[15,16]$. Therefore it is necessary to do compositional mapping of mixed cations systematically to determine the optimal ratio of counterions for high performance perovskite solar cells.

Inkjet printing of organic materials is a well-established technique in the organic electronic community [20,21]. In perovskite solar cells, inkjet printing [22,23] is relatively new and sparse. A majority of the "printing" research in perovskite solar cells involves screen-printing of mesoporous $\mathrm{TiO}_{2}$, and then the active layer is either drop-coated, dip-coated, or spin-coated on top of printed transport layer $[24,25]$. Very recently Wei et. al. have printed carbon based ink with organic cation on $\mathrm{PbI}_{2}$ film to fabricate perovskite solar cells [23]. Previous "printing" research in perovskite solar cells has not utilized the in situ mixing capabilities of an inkjet printer, and therefore combinations of reagents is limited to the physical number of ink cartridges [22-25]. Here we demonstrate the use of 
multi-channel printing of organic cations where the composition of the active layer can be easily and rapidly tuned by in situ mixing of cations from separate ink cartridges, for optimal performance and stability. Using this method virtually any ratio of counterions can be incorporated into the perovskite structure using a simple desktop inkjet printer, simply by assigning an RGB code on the computer software. Further, we have demonstrated that arrays of different perovskite structures can be prepared on the same substrate using multi-channel inkjet printing. Also, we show that using two channels in an inkjet printer to print MA and FA not only gives better control over compositional mapping, but also increases the reproducibility (small deviation in PCE device-to-device) by reducing experimental variation between substrates. Finally, we have found using this technique that devices prepared with a ratio of 2:1 MA to FA have the optimal performance. This strategy therefore enhances the possibility of combinatorial approach for better performing active layer perovskite materials.

\section{Experimental}

Lead tri-iodide-based perovskite solar cells were fabricated in air by sequential inkjet printing of alkyl-ammonium cations onto $\mathrm{PbI}_{2}$ thin film [1]. $\mathrm{PbI}_{2}$ films were first spin coated on pre-heated $\left(85^{\circ} \mathrm{C}\right)$ poly $(3,4-$-ethylenedioxythiophene) polystyrene sulfonate (PEDOT:PSS) coated ITO substrates from hot solution (400 $\mathrm{mg} \cdot \mathrm{mL}^{-1}$ concentration) of $\mathrm{PbI}_{2}$ in $N, N$-dimethylformamide (DMF) at 6000 r.p.m. for $35 \mathrm{~s}$. Films were then dried at $85^{\circ} \mathrm{C}$ for $30 \mathrm{~min}$. MA and FA cations were then printed from their respective solution in isopropanol $\left(40 \mathrm{mg} \cdot \mathrm{mL}^{-1}\right)$ onto $\mathrm{PbI}_{2}$ films using a multi-channel inkjet printer (Epson Artisan 50 Inkjet Printer) [26] where CYMK ink were replaced with the solution of either MA or FA (water was loaded in blank channels). The ratio of MA to FA was set based on the CYMK color combination (please refer to the ESI for detailed settings). As-cast films were then annealed at $90{ }^{\circ} \mathrm{C}$ for $30 \mathrm{~min}$. A thin layer of [6,6]-phenyl-C61-butyric acid methyl ester (PCBM) was then spin coated from a solution in chlorobenzene $\left(20 \mathrm{mg} \cdot \mathrm{mL}^{-1}\right.$ concentration $)$ at $1000 \mathrm{r} . \mathrm{p} . \mathrm{m}$. for $60 \mathrm{~s}$ inside $\mathrm{N}_{2}$ filled glove box. $15 \mathrm{~nm}$ of Ca followed by $100 \mathrm{~nm}$ of $\mathrm{Al}$ was thermally deposited at a chamber pressure of $1 \times 10^{-6}$ mbar. Current-voltage $(J-V)$ measurements were carried out inside $\mathrm{N}_{2}$ filled glove box under AM1.5G solar simulator at $100 \mathrm{~mW} \cdot \mathrm{cm}^{-2}$ light intensity.

\section{Results and discussion}


We characterized the as-prepared perovskite devices by scanning electron microscopy (SEM), atomic force microscopy (AFM) and powder x-ray diffraction (PXRD). SEM images in Fig. 1a-d show $100-500 \mathrm{~nm}$ crystals from different composition of MA to FA. The AFM images (Fig. 2b-f) of perovskite devices fabricated from various ratio of MA to FA show smooth film with RMS roughness of $25 \mathrm{~nm} \pm 6 \mathrm{~nm}$ (across all MA:FA ratios). The consistency in the film formation was confirmed by printing an array of devices in triplicate on the same substrates. An optical image of the array of devices shows varying composition of MA to FA in Fig. 2a.

The printed perovskite devices were structurally characterized using PXRD (Fig. 2g). PXRD spectra were normalized to their respective $\mathrm{PbI}_{2}(001)$ peaks at $2 \theta=12.6^{\circ}$ to show relative conversion to perovskite. The MA-only device shows a peak at $2 \theta=23.48^{\circ}$ (marked as *) which is attributed to the (211) peak of a tetragonal phase $(I 4 \mathrm{~cm})$. The FA-only device shows a peak at $2 \theta=11.7^{\circ}$ (marked as \#) which is attributed to the hexagonal phase [27] (100) peak, and a (101) peak at $2 \theta=13.9^{\circ}$ which is attributed to the trigonal (P3m1) phase [28]. The yellow hexagonal 'non-perovskite' phase is widebandgap and not applicable for photovoltaics. In the mixed counterion system, we only observed peaks attributable to the trigonal phase at $2 \theta \sim 14^{\circ}$. The average size of crystalline domains was $\sim 145$ $\mathrm{nm} \pm 21 \mathrm{~nm}$ (across all MA:FA ratios) as calculated by the Scherrer equation using a shape factor $K=$ 0.9. We observed a small peak attributable to $\mathrm{PbI}_{2}(001)$ peak at $2 \theta=12.6$. The ratio of $\mathrm{PbI}_{2}$ to $3 \mathrm{D}-$ perovskite from PXRD peaks at $2 \theta=12.6^{\circ}$ and $2 \theta \sim 14^{\circ}$ respectively was $1: 8.9,1: 7.5,1: 5.3,1: 3.9$, and 1:0.3 for MAI:FAI 1:0, 2:1, 1:1, 1:2, and 0:1 respectively. This pattern shows that the relative conversion of $\mathrm{PbI}_{2}$ to perovskite decreases with increasing concentration of FAI to MAI. Interestingly, it has been previously demonstrated that a small amount of $\mathrm{PbI}_{2}$ is necessary to reduce bulk recombination rate at the grain boundaries [29]. We have also observed an increasing $d$-spacing (from $6.28 \AA$ to $6.37 \AA$ ) in the $2 \theta \sim 14^{\circ}(002)(100)$ peak in the tetragonal phase or the (101) peak in the trigonal phase with the increasing FA concentration as shown in Fig. 3.

We have measured the device efficiency under AM1.5G solar simulator at $100 \mathrm{mWcm}^{-2}$ light intensity by sweeping the voltage from forward to reverse direction at a scan rate of $250 \mathrm{mV} \cdot \mathrm{s}^{-1}$. A small hysteresis was observed in these samples. We found that 2:1 ratio of MA to FA has a higher 
PCE compared to other compositions, consistent with the results obtained by Pellet et al [16]. The device performances are listed in Table 1. The highest PCE achieved was $11.1 \%$ with a current density $J_{\mathrm{SC}}=18.77 \mathrm{~mA} \cdot \mathrm{cm}^{-2}$, Open circuit voltage $V_{\mathrm{OC}}=0.87 \mathrm{~V}$ and fill factor $\mathrm{FF}=68 \%$ (Fig. 4). Significantly low PCE observed in FA-only device is due to the presence of hexagonal phase and poor conversion to perovskite (consistent with weak perovskite peak at $2 \theta \sim 14^{\circ}$ ).

\section{Conclusions}

In conclusion, we have demonstrated that the inkjet printing of organic cations on spin-coated lead iodide films opens up opportunities for high throughput screening of cations, showing great promise in the creation of perovskite solar cells. Structural, optical and electronic properties of perovskite materials can be systematically tuned by simply mixing multiple cations via multi-channel inkjet printing. This technique will also reduce the irreproducibility observed in perovskite formation during sequential processing; this is evident in the 2:1 MA to FA device, where the standard deviation in measured PCE is only $\pm 0.3 \%$. Inkjet printing of counterions gives smooth films with high percentage of lead iodide to perovskite conversion. We have achieved device efficiency comparable to spin coated film. Such technique is also potentially compatible with roll-to-roll fabrication for industrial purpose.

\section{Acknowledgements}

This work was supported as part of Polymer-Based Materials for Harvesting Solar Energy, an Energy Frontier Research Centre funded by the U.S. Department of Energy, Office of Science, Basic Energy Sciences under Award \#DE-SC0001087. V.M.R. acknowledges support from the NSF (CHE$1307021)$.

\section{References}

[1] Burschka J, Pellet N, Moon S-J, Humphry-Baker R, Gao P, Nazeeruddin MK, et al. Sequential deposition as a route to high-performance perovskite-sensitized solar cells. Nature. 2013;499:316-9.

[2] Etgar L, Gao P, Xue Z, Peng Q, Chandiran AK, Liu B, et al. Mesoscopic CH3NH3PbI3/TiO2 Heterojunction Solar Cells. J Am Chem Soc. 2012;134:17396-9.

[3] Green MA, Ho-Baillie A, Snaith HJ. The emergence of perovskite solar cells. Nature Photon. 2014;8:506-14.

[4] McGehee MD. PEROVSKITE SOLAR CELLS Continuing to soar. Nat Mater. 2014;13:845-6. 
[5] Nie W, Tsai H, Asadpour R, Blancon J-C, Neukirch AJ, Gupta G, et al. High-efficiency solution-processed perovskite solar cells with millimeter-scale grains. Science. 2015;347:522-5.

[6] Lee MM, Teuscher J, Miyasaka T, Murakami TN, Snaith HJ. Efficient Hybrid Solar Cells Based on MesoSuperstructured Organometal Halide Perovskites. Science. 2012;338:643-7.

[7] Zhou H, Chen Q, Li G, Luo S, Song T-b, Duan H-S, et al. Interface engineering of highly efficient perovskite solar cells. Science. 2014;345:542-6.

[8] You J, Hong Z, Yang Y, Chen Q, Cai M, Song T-B, et al. Low-Temperature Solution-Processed Perovskite Solar Cells with High Efficiency and Flexibility. ACS Nano. 2014;8:1674-80.

[9] Jeon NJ, Noh JH, Kim YC, Yang WS, Ryu S, Seok SI. Solvent engineering for high-performance inorganic-organic hybrid perovskite solar cells. Nat Mater. 2014;13:897-903.

[10] Seo J, Park S, Chan Kim Y, Jeon NJ, Noh JH, Yoon SC, et al. Benefits of very thin PCBM and LiF layers for solution-processed p-i-n perovskite solar cells. Energy Environ Sci. 2014;7:2642-6.

[11] Dong H, Wu Z, Xia B, Xi J, Yuan F, Ning S, et al. Modified deposition process of electron transport layer for efficient inverted planar perovskite solar cells. Chem Commun. 2015;51:8986-9.

[12] Borriello I, Cantele G, Ninno D. Ab initio investigation of hybrid organic-inorganic perovskites based on tin halides. Phys Rev B. 2008;77:235214.

[13] Knutson JL, Martin JD, Mitzi DB. Tuning the Band Gap in Hybrid Tin Iodide Perovskite Semiconductors Using Structural Templating. Inorg Chem. 2005;44:4699-705.

[14] Koh TM, Fu K, Fang Y, Chen S, Sum TC, Mathews N, et al. Formamidinium-Containing Metal-Halide: An Alternative Material for Near-IR Absorption Perovskite Solar Cells. J Phys Chem C. 2013;118:16458-62.

[15] Aharon S, Dymshits A, Rotem A, Etgar L. Temperature dependence of hole conductor free formamidinium lead iodide perovskite based solar cells. J Mater Chem A. 2015;3:9171-8.

[16] Pellet N, Gao P, Gregori G, Yang T-Y, Nazeeruddin MK, Maier J, et al. Mixed-Organic-Cation Perovskite Photovoltaics for Enhanced Solar-Light Harvesting. Angew Chem Int Ed. 2014;53:3151-7.

[17] Filip MR, Eperon GE, Snaith HJ, Giustino F. Steric engineering of metal-halide perovskites with tunable optical band gaps. Nat Commun. 2014;5:5757.

[18] Sanchez RS, Gonzalez-Pedro V, Lee J-W, Park N-G, Kang YS, Mora-Sero I, et al. Slow Dynamic Processes in Lead Halide Perovskite Solar Cells. Characteristic Times and Hysteresis. J Phys Chem Lett. 2014;5:2357-63.

[19] Eperon GE, Stranks SD, Menelaou C, Johnston MB, Herz LM, Snaith HJ. Formamidinium lead trihalide: a broadly tunable perovskite for efficient planar heterojunction solar cells. Energy Environ Sci. 2014;7:982-8. 
[20] Calvert P. Inkjet Printing for Materials and Devices. Chem Mater. 2001;13:3299-305.

[21] Teichler A, Perelaer J, Schubert US. Inkjet printing of organic electronics - comparison of deposition techniques and state-of-the-art developments. J Mater Chem C. 2013;1:1910-25.

[22] Li S-G, Jiang K-J, Su M-J, Cui X-P, Huang J-H, Zhang Q-Q, et al. Inkjet printing of CH3NH3PbI3 on a mesoscopic TiO2 film for highly efficient perovskite solar cells. J Mater Chem A. 2015;3:9092-7.

[23] Wei Z, Chen H, Yan K, Yang S. Inkjet Printing and Instant Chemical Transformation of a CH3NH3PbI3/Nanocarbon Electrode and Interface for Planar Perovskite Solar Cells. Angew Chem. 2014;126:13455-9.

[24] Hu M, Liu L, Mei A, Yang Y, Liu T, Han H. Efficient hole-conductor-free, fully printable mesoscopic perovskite solar cells with a broad light harvester NH2CH=NH2PbI3. J Mater Chem A. 2014;2:17115-21.

[25] Liu L, Mei A, Liu T, Jiang P, Sheng Y, Zhang L, et al. Fully Printable Mesoscopic Perovskite Solar Cells with Organic Silane Self-Assembled Monolayer. J Am Chem Soc. 2015;137:1790-3.

[26] Creran B, Yan B, Moyano DF, Gilbert MM, Vachet RW, Rotello VM. Laser desorption ionization mass spectrometric imaging of mass barcoded gold nanoparticles for security applications. Chem Commun. 2012;48:4543-5.

[27] Stoumpos CC, Malliakas CD, Kanatzidis MG. Semiconducting Tin and Lead Iodide Perovskites with Organic Cations: Phase Transitions, High Mobilities, and Near-Infrared Photoluminescent Properties. Inorg Chem. 2013;52:901938.

[28] Baikie T, Fang Y, Kadro JM, Schreyer M, Wei F, Mhaisalkar SG, et al. Synthesis and crystal chemistry of the hybrid perovskite (CH3NH3)PbI3 for solid-state sensitised solar cell applications. J Mater Chem A. 2013;1:5628-41.

[29] Chen Q, Zhou H, Song T-B, Luo S, Hong Z, Duan H-S, et al. Controllable Self-Induced Passivation of Hybrid Lead Iodide Perovskites toward High Performance Solar Cells. Nano Lett. 2014;14:4158-63.

\section{Figure captions}

Fig. 1. (a-d) SEM image of perovskite samples fabricated by inkjet printing of 1:0, 2:1, 1:1, and 0:1 MAI and FAI respectively on $\mathrm{PbI}_{2}$ coated substrate at various ratio. Inset: magnified SEM of the same substrate.

Fig. 2. (a) Optical image, (b-f) AFM image of 1:0, 2:1, 1:1, and 0:1 MAI and FAI respectively (the black scale bar is $2 \mu \mathrm{m}$ ) and (g) PXRD spectra of perovskites where organic cations MA and FA were inkjet-printed from isopropanol. The mixing of two cations was done in-situ on the $\mathrm{PbI}_{2}$ coated substrates. PXRD spectra are normalized by their respective $\mathrm{PbI}_{2}(001)$ peaks at $2 \theta=12.6^{\circ}$ to show relative conversion to perovskite.

Fig. 3. PXRD peaks of perovskites at various composition ratio of MA to FA fabricated from multi-channel inkjet printing of cations on spin-coated $\mathrm{PbI}_{2}$ substrates. 
Fig. 4. Current-voltage curve of a perovskite solar cell fabricated from 2:1 MA to FA printed on spin-coated $\mathrm{PbI}_{2}$ film.

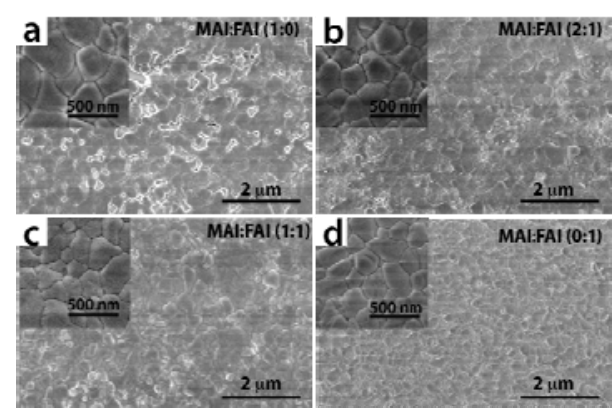

Fig. 1.

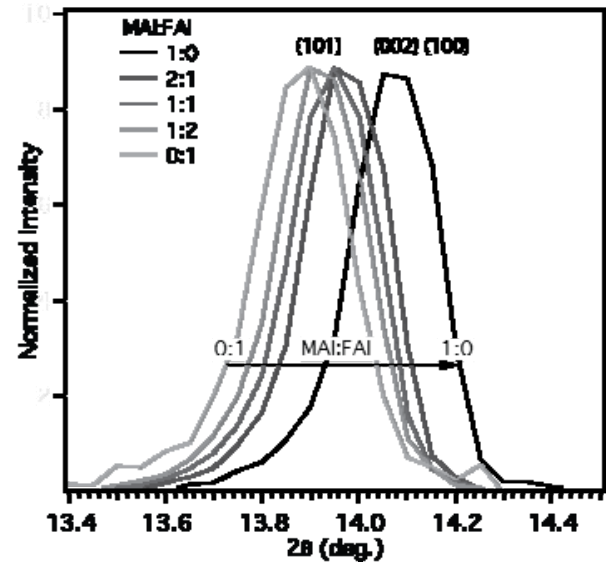

Fig. 3
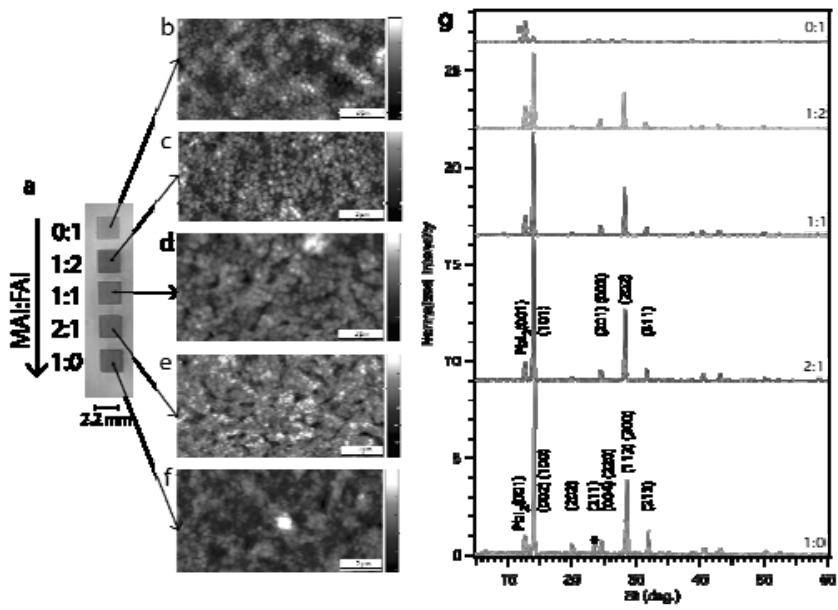

Fig. 2

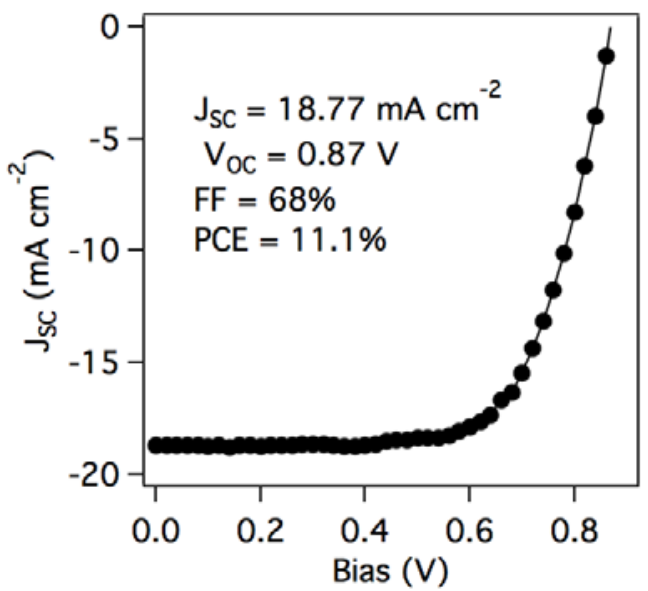

Fig. 4

Table 1. Perovskite device performance parameters at different composition ratio of MA to FA as organic cations.

\begin{tabular}{lllll}
\hline MAI:FAI & $V_{\mathrm{OC}}(\mathrm{mV})$ & $J_{\mathrm{SC}}\left(\mathrm{mA} \cdot \mathrm{cm}^{-2}\right)$ & FF $(\%)$ & PCE $(\%)$ \\
\hline $1: 0$ & $917 \pm 11$ & $14.3 \pm 0.6$ & $67 \pm 1$ & $8.7 \pm 0.4$ \\
$2: 1$ & $866 \pm 5$ & $18.7 \pm 0.2$ & $66 \pm 1$ & $10.8 \pm 0.3$ \\
$1: 1$ & $932 \pm 22$ & $13.7 \pm 1.0$ & $70 \pm 5$ & $8.8 \pm 0.2$ \\
$1: 2$ & $912 \pm 5$ & $12.4 \pm 1.9$ & $68 \pm 2$ & $7.6 \pm 1.3$ \\
$0: 1$ & $725 \pm 190$ & $3.5 \pm 1.1$ & $51 \pm 8$ & $1.2 \pm 0.2$ \\
\hline
\end{tabular}

